Richter, Ingo

\title{
Öffentliche Verantwortung für das Aufwachsen von Kindern und
} Jugendlichen

Diskurs 12 (2002) 1, S. 11-16

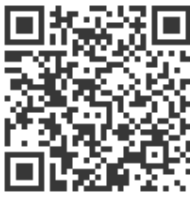

Quellenangabe/ Reference:

Richter, Ingo: Öffentliche Verantwortung für das Aufwachsen von Kindern und Jugendlichen - In:

Diskurs 12 (2002) 1, S. 11-16 - URN: urn:nbn:de:0111-pedocs-106964 - DOI: 10.25656/01:10696

https://nbn-resolving.org/urn:nbn:de:0111-pedocs-106964

https://doi.org/10.25656/01:10696

in Kooperation mit / in cooperation with:
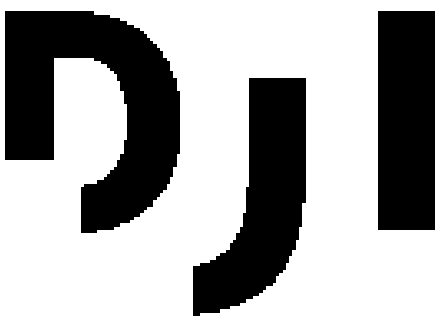

\section{Deutsches Jugendinstitut}

www.dji.de/diskurs

\section{Nutzungsbedingungen}

Gewährt wird ein nicht exklusives, nicht übertragbares, persönliches und beschränktes Recht auf Nutzung dieses Dokuments. Dieses Dokument ist ausschließlich für den persönlichen, nicht-kommerziellen Gebrauch bestimmt. Die Nutzung stellt keine Übertragung des Eigentumsrechts an diesem Dokument dar und gilt vorbehaltlich der folgenden Einschränkungen: Auf sämtlichen Kopien dieses Dokuments müssen alle Urheberrechtshinweise und sonstigen Hinweise auf gesetzlichen Schutz beibehalten werden. Sie dürfen dieses Dokument nicht in irgendeiner Weise abändern, noch dürfen Sie dieses Dokument für öffentliche oder kommerzielle Zwecke vervielfältigen, öffentlich ausstellen, aufführen, vertreiben oder anderweitig nutzen.

Mit der Verwendung dieses Dokuments erkennen Sie die Nutzungsbedingungen an

\section{Terms of use}

We grant a non-exclusive, non-transferable, individual and limited right to using this document.

This document is solely intended for your personal, non-commercial use. Use of this document does not include any transfer of property rights and it is conditional to the following limitations: All of the copies of this documents must retain all copyright information and other information regarding legal protection. You are not allowed to alter this document in any way, to copy it for public or commercial purposes, to exhibit the document in public, to perform, distribute or otherwise use the document in public.

By using this particular document, you accept the above-stated conditions of use.

\section{Kontakt / Contact:}

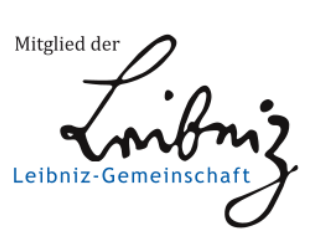

DIPF | Leibniz-Institut für Bildungsforschung und Bildungsinformation Informationszentrum (IZ) Bildung

E-Mail: pedocs@dipf.de

Internet: www.pedocs.de 


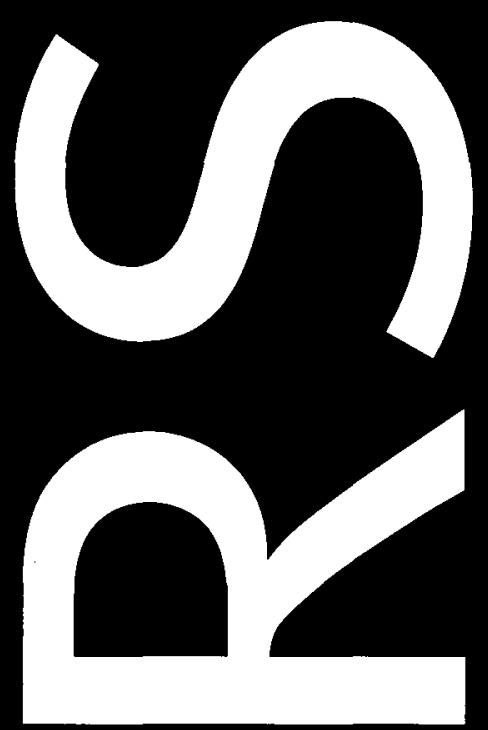

\section{Thema}

\section{Öffentliche Verantwortung} und soziale Gerechtigkeit Verhandlungen um das Gemeinwohl

\section{Spektrum}

- Mediale Großereignisse als kulturelle Sozialisation

- Die Karriere des Gender Mainstreaming in Politik und Wissenschaft

- Junge Lesben und Schwule - zwischen Heteronormativität und posttraditioneller Vergesellschaftung

Trends

- Die Bürgergesellschaft - auch ein Forschungsprogramm? 


\title{
Öffentliche Verantwortung und soziale Gerechtigkeit - Verhandlungen um das Gemeinwohl
}

\author{
Jutta Müller-Stackebrandt / Christian Lüders \\ Öffentliche Verantwortung und Gerechtigkeit - zwei \\ Leitbegriffe des Elften Kinder- und Jugendberichts
}

\author{
Ingo Richter \\ Öffentliche Verantwortung für das Aufwachsen \\ von Kindern und Jugendlichen
}

Trotz allen Geredes von der "Entstaatlichung des Staates « ist daran festzuhalten, dass der Staat die öffentliche Verantwortung für das Aufwachsen von Kindern und Jugendlichen zu gewährleisten hat. Im traditionellen Sozialstaat musste der Staat das sogenannte Existenzminimum der Menschen garantieren. Öffentliche Verantwortung heißt dagegen, dass der Staat eine Selbsthilfefähigkeit sicherstellen - und schlimmstenfalls zahlen - muss.

\section{Annemarie Gerzer-Sass}

\section{Der Beitrag der Wirtschaft zum Aufwachsen} in öffentlicher Verantwortung

In Deutschland wird eine familienfreundiche Arbeitswelt in weiten Kreisen der Bevölkerung und der Öffentlichkeit immer noch als Teil der Sozial- und Frauenpolitik begriffen. Die Frage der Verbindung von Familie und Beruf ist aber komplexer: So geht es vor allem auch darum, wie die Rolle des Mannes aus ihrer funktional einseitigen Definition als Haupternährer und "Berufsmensch « herausgelöst werden kann. Dazu bedarf es nicht nur eines individuellen »Umerziehungsprozesses", sondern vielmehr eines kulturellen Wandels, der vor allem auch von Unternehmen getragen werden muss.

Holger Lengfeld

Soziale Gerechtigkeit und politische Entscheidungen Perspektiven der interdisziplinären

Gerechtigkeitsforschung

Soziale Gerechtigkeit ist ein Begriff, der im politischen Alltag zwar häufig bemüht wird, aber leicht in den Geruch politischer Rhetorik gerät. Soziale Gerechtigkeit - mithin nichts anderes als ein schmückendes Beiwerk politischer Entscheidungen, das der Politik deshalb keinen verlässlichen Maßstab bereitstellen kann, weil ein jeder darunter etwas anderes versteht? Befunde der empirischen Gerechtigkeitsforschung lassen erkennen, dass Gerechtigkeitserwägungen durchaus zur Qualifizierung politischer Entscheidungen beitragen könnten. 
Jürgen Zinnecker

Mediale Großereignisse als kulturelle Sozialisation das Beispiel des 11. Septembers

Eine aktuelle Befragungsstudie des Siegener Zentrums für Kindheit-, Jugend- und Biografieforschung bietet Gelegenheit, die Verarbeitung medialer Großereignisse durch Heranwachsende mit Mitteln der empirischen Sozialforschung nachzuzeichnen. Während die Kinder und Jugendlichen befragt wurden, ereigneten sich die Terroranschläge in den USA. Wie würde sich die Stimmungslage der Kinder und Jugendlichen durch dieses Großereignis verändern, das sie fast alle live am Fernseher zu Hause verfolgt hatten?

\section{Sigrid Metz-Göckel}

\section{Die Karriere des Gender Mainstreaming in Politik und Wissenschaft}

Etikettenschwindel oder neuer Schritt im Geschlechterund Generationenverhältnis?

In den letzten Jahren hat sich in Politik und Wissenschaft eine neue Begrifflichkeit breit gemacht, die Frauen sprachlich wieder unsichtbar macht: Gender Mainstreaming. Was ist dran an der rasanten Karriere dieser Begriffsbildung - was ist Sache, was Problem, was nur Etikettenschwindel? Anhand von Befunden zweier empirischer Studien zur Umsetzung des Gender Mainstreaming werden Chancen und Gefahren dieses Konzepts abgewogen.

\section{Sabine Hark}

\section{Junge Lesben und Schwule - zwischen Heteronormativität} und posttraditionaler Vergesellschaftung

s/ch habe nichts gegen Lesben und Schwule - solange sie mir nicht zu nahe treten, solange sie nicht öffentlich auftreten." Unterliegen junge Lesben und Schwule dem sheimlichen « Gebot, heimlich zu bleiben? Es scheint, dass nicht so sehr die Differenz in der sexuellen Objektwahl oder vermeintlich andere sexuelle Praktiken die Ursache für die Diskriminierung von Schwulen und Lesben darstellen, sondern die unterstellte Abweichung von den akzeptierten Formen von Männlich- und Weiblichkeit.

\section{Thomas Rauschenbach}

Die Bürgergesellschaft - auch ein Forschungsprogramm? Anmerkungen zum Projekt der Bürgergesellschaft und zum Bedarf an Forschung

Warum bedarf eigentlich ein so dezidiert politisches Projekt wie das der Bürgergesellschaft vermehrter Forschung? Der Hauptgrund liegt darin, dass dieses Vorhaben so anspruchsvoll ist, dass es entweder nur ständig gefordert, erwünscht, erdacht werden kann, oder aber in seinen Prämissen und seinen Effekten genauer geklärt und geprüft werden muss. Empirische Forschung kann eine Brücke sein, um auch theoretisch gehaltvoller über mögliche Chancen, Risiken und $\mathrm{Ne}$ benwirkungen der Bürgergesellschaft diskutieren zu können. 


\section{Ingo Richter}

\section{Öffentliche Verantwortung für das Aufwachsen von Kindern und Jugendlichen ${ }^{1}$}

Prof. Dr. Ingo Richter, geb. 1938, Honorarprofessor an der Universität Tübingen; von 1993 bis Juli 2002 Direktor und Vorstand des Deutschen Jugendinstituts e. V. (DJI), München; Herausgeber der Zeitschrift

"Recht der Jugend und des Bildungswesens«. Arbeitsschwerpunkte: Bildungspolitik und Bildungsrecht, insbesondere Schulrecht, Recht der Weiterbildung; Sozialpolitik und Sozialrecht, insbesondere Jugend- und Jugendhilferecht; Verfassungsrecht.

Neuere Veröffentlichungen: Die sieben Todsünden der Bildungspolitik. München 1999; (hrsg. zus. mit Andreas Flitner und Christian Petry) Wege aus der Ausbildungskrise, Memorandum des Forums "Jugend. Bildung. Arbeit«. Opladen 1999; (hrsg. zus. mit Sabine Sardei-Biermann) Jugendarbeitslosigkeit. Ausbildungs- und Beschäftigungsprogram. me in Europa. Opladen 2000

Tengstraße 22

D-80798 München
Der Begriff »öffentliche Verantwortung « - Leitmotiv des Elften Kinder- und Jugendberichts - wird in dem Beitrag entlang zentraler Merkmale und Handlungselemente entfaltet. Hauptanliegen des Autors ist es, das Aufwachsen von Kindern und Jugendlichen jenseits der traditionellen Gegenüberstellung von Eltern und Staat zu thematisieren. Gründe für eine Neuformulierung dieser Thematik sieht der Autor in der Entwicklung des Sozialstaats: Individuen, Familien und soziale Gruppen werden zukünftig größere Eigenverantwortung für ihre Lebensbedingungen tragen müssen. An die Stelle des Sozialstaats, der die soziale Sicherheit vor allem durch monetäre Transferleistungen zu gewährleisten meinte, tritt nun die öffentliche Verantwortung als Voraussetzung, dass die Menschen eigenverantwortlich ihre Probleme lösen können. Nach einem Durchgang durch unterschiedliche Träger öffentlicher Verantwortung in der pluralistischen Gesellschaft und einer allgemeinen Begründung der Kinderund Jugendpolitik als "Lebenslagenpolitik « bilanziert der Autor öffentliche Verantwortung durch staatliche Tätigkeit sowie entlang der Gewährleistung von Selbsthilfefähigkeit der gesellschaftlichen Akteure.

\section{Was heißt Verantwortung?}

\section{Die Baustelle}

Sie kennen alle das gelbe Schild, das an Baustellen angebracht ist: "Vorsicht Baustelle. Betreten verboten. Eltern haften für ihre Kinder«. Die 7-jährigen Knaben A und B sind auf dem Heimweg von der Schule und kommen an einer gesicherten, zurzeit verlassenen Baustelle vorbei, an der 
das genannte Schild angebracht ist. Durch ein Loch im Bauzaun dringen sie in das Baugelände ein und turnen eine Weile auf den Gerüsten herum. Hierbei lockert sich ein an sich fachgerecht angebrachtes Brett so, dass es nur noch wenig aufliegt. $A$ und $B$ und kriechen wieder durch das Loch im Bauzaun zurück, wobei sich $A$ am Drahtgeflecht verletzt und mangels einer Tetanusimpfung wegen drohenden Wundbrandes ärztlich behandelt werden muss. Nur eine Stunde später kriechen die 11-jährigen Knaben $C$ und $D$, ebenfalls auf dem Heimweg von der Schule, auch durch das Loch im Bauzaun und turnen auf dem Gerüst herum, wobei D wegen des unsicheren Brettes vom Gerüst stürzt und sich schwer verletzt. - Ein Fall, den das Leben schrieb!

\section{Rauchen und Lungenkrebs}

Es ist völlig unbestritten, dass es eine hohe positive Korrelation zwischen Tabakkonsum und dem Auftreten von Lungenkrebs gibt. Empirische Untersuchungen, die auch die Elfte Kinder- und Jugendberichtskommission in ihrem Bericht referiert, zeigen, dass junge Menschen in großem Umfang rauchen, wobei es im Laufe der Zeit Verschiebungen zwischen den unterschiedlichen Jugendpopulationen gegeben hat. Der Deutsche Kinderschutzbund erhebt nach US-amerikanischem Vorbild - Klage gegen die Unternehmen der Tabakindustrie mit dem Antrag, 50 Millionen Euro für die Errichtung und Unterhaltung von Entziehungseinrichtungen und Lungenheilstätten zurVerfügung zu stellen. Die Eltern von mehreren minderjährigen Jugendlichen klagen gegen den Verband der Automatenaufsteller sowie gegen einzelne Automatenaufsteller mit dem Ziel, die Selbstbedienung von Minderjährigen an Zigarettenautomaten durch entsprechende technische Vorkehrungen zu verhindern. Die Bundestagsfraktion der Grünen schließlich verlangt im Namen aller in Deutschland lebenden Kinder und Jugendlichen von der Bundesregierung ein Verbot der Zigarettenwerbung im Kino, die sich gezielt an ein jugendliches Publikum wendet.

Diese zwei typischen Fälle könnten unter juristischen Gesichtspunkten analysiert werden, z. B. könnte beim ersten Fall gefragt werden, wer oder wessen Versicherung die Kosten für die ärztliche Behandlung zu tragen oder eventuell Schmerzensgeld zu zahlen hätte? Ob die Versicherung Rückgriff nehmen würde, und zwar gegen wen? Ob sich jemand vielleicht sogar strafbar gemacht hat?

Auch im zweiten Fall könnte nach den juristischen Voraussetzungen und Chancen solcher Initiativen, Schadensersatz zu erstreiten, gefragt werden. Hier interessiert aber der Aspekt der Verantwortung. Es geht darum, wer (Subjekt) in welcher Eigenschaft (Trägerschaft) für wen (Objekt) in welcher Zeit (Zeitraum) und welcher Form (Form) Verantwortung zu tragen hat?
- Als Subjekt hätten im ersten Fall die Eltern ganz individuell Verantwortung zu tragen, im zweiten Fall wären es kollektiv die Unternehmen, Verbände, Organe.

- Als Verantwortungsträger haften die Eltern vollkommen privat (Trägerschaft), während es sich bei den Unternehmen oder Staatsorganen bisher eher um öffentliche Kosten handelt, die in Anspruch genommen werden.

- Die Objekte, für die individuell Verantwortung übernommen wird, sind im ersten Fall die ärztlichen Kosten der einzelnen Kinder, während es im Raucherfall kollektiv die Gesundheitsrisiken aller Raucher sind.

Der Zeitraum der Ereignisse ist verschieden: Im Baustellenfall geht es um ein Ereignis in der Vergangenheit, beim Rauchen geht es um mögliche Folgen in der Zukunft.

- Die Form der auftretenden Schädigung ist im Falle der Baustelle sehr konkret, während das Gesundheitsrisiko der Raucher einstweilen abstrakt bleibt.

Das Einzige, was beide Fälle miteinander trotz ihrer Verschiedenartigkeit verbindet, ist der Begriff der Verantwortung. Was hätten die zwei unterschiedlichen Akteure und Verantwortungsträger jeweils tun können, um Schadensfälle zu vermeiden? Was heißt Verantwortung in diesem Zusammenhang und worin besteht eigentlich "Verantwortung tragen « praktisch, im Alltag?

Was hätten die Eltern eigentlich im Baustellenfall tun sollen, um die Unfälle zu verhindern? Hätten sie

- die Kinder über die Risiken des Schulwegs aufklären sollen? (Erziehung)

- die Kinder nicht zur Schule schicken sollen, so lange das Loch im Bauzaun ist? (Prohibition)

- den Kindern das Betreten der Baustelle untersagen sollen? (Verbote)

- die Kindervor der Baustelle warnen sollen? (Prävention)

- die Kinder auf dem Schulweg begleiten sollen? (Aufsicht) und/oder

- die Kinder im Nachhinein bestrafen sollen? (Sanktionen)

Was würde von der Tabakindustrie, den Automatenaufstellern, der Bundesregierung im zweiten Fallbeispiel erwartet, um Kinder und Jugendliche zu schützen? - die Einstellung der Zigarettenherstellung bzw. des Rauchens durch die Erwachsenen? (Unterlassen) - das Verbot der Zigarettenherstellung? (Repression)

- die Reduzierung der Zigarettenherstellung? (Selbstbeschränkung)

- keinen Verkauf an Kinder und Jugendliche? Oder zumindest keinen Automatenverkauf? (Vertriebsbeschränkungen)

- die Sicherung der Aufbewahrung? (Zugangsbeschränkung)

- generelle oder spezielle Rauchverbote, gegebenenfalls mit Strafsanktionen? (Verhaltensregulierung)

- Warnung vor dem Zigarettenkonsum und Werbebeschränkung? (Prävention) 
- Werbung für eine gesunde Lebensweise - "stark und schön ohne Tabak«? (positive Konditionierung)

Es gibt also theoretisch ein umfassendes Repertoire des Verhaltens in »Verantwortungs «-Situationen und ganz verschiedene Facetten der Verantwortungsträgerschaft. Aber nicht alle Handlungsmöglichkeiten sind zugelassen oder machbar: So gibt es die Freiheit der Selbstschädigung - zumindest für Erwachsene. Wichtig ist hier jedoch Folgendes: Im ersten Fall handelt es sich um die persönliche, individuelle und private Verantwortung der Eltern bzw. Erziehungsberechtigten für ihre unmündigen Kinder und im zweiten Fall um die kollektive, abstrakte und öffentliche Verantwortung "der « Gesellschaft, bzw. von Organisationen, Verbänden und Staat.

\section{Der Begriff »Öffentliche Verantwortung«?}

In diesem Abschnitt wird der Begriff der öffentlichen Verantwortung - das Leitmotiv des Elften Kinder- und Jugendberichts erläutert. Im Elften Kinder- und Jugendbericht geht es dabei ausschließlich um eine Verantwortung, die - wie im zweiten Fallbeispiel - durch folgende Merkmale gekennzeichnet ist:

- Verantwortung für die Zukunft,

- abstrakte Verantwortung,

- kollektive Verantwortung,

- Verantwortung für eine Gesamtheit, die der Kinder und Jugendlichen nämlich, eine "öffentliche Verantwortung «.

Diese Form der Verantwortung, die öffentliche Verantwortung für das Aufwachsen von Kindern und Jugendlichen, hat in einem spezifischen Sinn mit der Elternverantwortung zu tun. Die öffentliche Verantwortung für das Aufwachsen von Kindern und Jugendlichen schließt nämlich die Elternverantwortung nicht aus - ganz im Gegenteil: Sie setzt sie voraus und soll sie ermöglichen und stärken. Die Diskussion über die Verantwortung für das Aufwach. sen von Kindern und Jugendlichen wird durch die grundlegende Position der Eltern und des Staates bestimmt, und zwar durch das Elternrecht (Art. 6 Abs. 2 Satz 1 Grundgesetz (GG)) und das so genannte staatliche Wächteramt (Art. 6 Abs. 2 Satz 2 GG), das Recht der Kinder und Jugendlichen auf Erziehung und Bildung (Art. 2 Abs. $1 \mathrm{GG}, \S 1$ Kinder- und Jugendhilfegesetz (KJHG) und die Schulgesetze der Länder) und seine Erfüllung durch die Kinder- und Jugendhilfe bzw. das Schulwesen, beides unter staatlicher Aufsicht. Daraus folgt: Die Kommission Elfter Kinder- und Jugendbericht fordert eine öffentliche Verantwortung für das Aufwachsen von Kindern und Jugendlichen, damit die Eltern ihre Kinder erziehen können.

Es ist ein groteskes oder bösartiges Missverständnis, Elternrecht und öffentliche Verantwortung als Nullsummenspiel zu betrachten: »Was die eine Seite verliert, gewinnt die andere! « - wie eine Glosse der FAZ vom 20.07.01, gleich nach der Übergabe des Berichtes an das zuständige Bundesministerium, ohne jede Kenntnis des Berichts selber - dieser war zur damaligen Zeit noch gar nicht zugänglich - unterstellte:

\section{"Kinderverstaatlichung}

Was Kinder vor allem anderen brauchen, weiß jeder: Sie brauchen Liebe, Geborgenheit, Zeit. Was davon kann der Staat ihnen geben? Nichts. Über nichts hat aber die von Jugendministerin Bergmann eingesetzte Sachverständigenkommission nicht 600 Seiten geschrieben. Der Elfte Kinder-und Jugendbericht der Bundesregierung handelt vielmehr von dem, was Eltern wünschen, um sich Kinder finanziell und zeitlich leisten zu können: staatliche Rundumbetreuung von der Wiege bis zum Discoalter. Von den Verfassern des Berichts war nicht zu erwarten, dass sie in dieser Entwicklung auch eine der Ursachen oft beklagter Probleme der Jugend suchen würden. Das liefe der mittlerweile von allen Parteien getragenen Kinderverstaatlichung diametral entgegen. Schon der Titel des Berichts verheißt, 
dass auf diesem Weg munter vorangeschritten wird: >Aufwachsen in öffentlicher Verantwortung ‘. Da greift eine kalte Hand nach den Kleinsten. Vermessen ist, wer von einem Jugendbericht auch Einsichten wie diese erwartet: Noch so gut geführte Krippen und Horte können Kindern nicht das Gefühl vermitteln, um ihrer selbst willen geliebt zu werden; noch so ausgedehnte Betreuungszeiten nicht die Gewissheit, dass immer jemand für sie da ist. Der Staat kann nicht Vorbild sein und auch die Erziehung nicht ersetzen.»

Vielleicht sollte der Verantwortungsbegriff in diesem Zusammenhang durch seine Handlungselemente näher umschrieben werden. Verantwortung heißt:

- Aufgaben erkennen,

- Pflichten erfüllen,

- Rechenschaft ablegen,

- für Menschen einstehen und - nicht zuletzt -

- für Versagen haften.

Insbesondere dieser letzte Punkt, wonach Versagen bei der Ausübung von Verantwortung Folgen hat, kann nicht genug betont werden. Für die Eltern im Baustellenfall sind die möglichen Folgen klar; aber wie steht es mit den Folgen für die Tabakindustrie, die Automatenaufsteller, die Bundesregierung? Können diese auch versagen? Und wenn ja, was passiert dann?

Um diese Frage beantworten zu können, muss man sich den Grund für die Übernahme von Verantwortung deutlich machen: Im ersten Fall ist der allgemeine Grund für die Elternverantwortung klar: Es ist die Pflicht zur elterlichen Sorge, Elternrecht und Elternpflicht (Art. 6 Abs. 2 GG und $\$ 1626$ Bürgerliches Gesetzbuch (BGB)). Wie aber steht es im zweiten Fall mit dem Grund für die Verantwortung? Warum trägt die Tabakindustrie Verantwortung für den Lungenkrebs der Raucher? Weil sie die Zigaretten herstellt? Und die Automatenaufsteller? Weil sie die Zigaretten - auch Kindern und Jugendlichen zugänglich machen? Weil beide mit der Schaffung der Risiken Geld verdienen? Tragen nicht die Raucherinnen und Raucher selber die Verantwortung für ihre Selbstschädigung? Schließlich werden sie von den Herstellern und von der Bundesregierung gewarnt. Können auch Kinder und Jugendliche diese Verantwortung selber tragen? Und schließlich die Bundesregierung? Warum soll sie die Verantwortung dafür tragen, dass die Menschen aufgrund von Selbstschädigung früher sterben? Es gibt ein Grundrecht auf Leben und auf Gesundheit (Art. 2 Abs. 2 GG), und der Sozialstaat trägt nach unserer Verfassung die
Verantwortung, dass die Menschen von ihren Grundrechten auch Gebrauch machen können. Was nützen die schönsten Grundrechte, wenn niemand von ihnen Gebrauch machen kann?

Diese oben aufgeführten fünf Handlungselemente werden am Schluss noch einmal aufgegriffen werden. Zuvor soll jedoch ein Blick auf den Begriff der öffentlichen Verantwortung geworfen werden, so wie er im Elften Kinder- und Jugendbericht eingeführt wird.

\section{„Öffentliche Verantwortung« im Elften Kinder- und Jugendbericht}

Von einer öffentlichen Verantwortung zu sprechen heißt wie schon erwähnt - , das Aufwachsen von Kindern und Jugendlichen jenseits der traditionellen Gegenüberstellung von Eltern und Staat zu thematisieren.

Der Grund für die Neuformulierung dieser Themen ist die Entwicklung des Sozialstaates. Auch wenn man die Begründungen für den quantitativen Abbau sozialstaatlicher Leistungen und den qualitativen Umbau sozialstaatlicher Organisations- und Verfahrensformen kritisiert, wie es die Elfte Kinder- und Jugendberichtskommission in ihrem Bericht tut, kann man nicht darüber hinwegsehen, dass die Individuen, die Familien und die sozialen Gruppen in Zukunft eine größere Selbständigkeit und Eigenverantwortung für ihre Lebensbedingungen tragen werden, 
als dies im »lahrhundert des Sozialstaates«, im 20. Jahrhundert, der Fall gewesen ist. Mit anderen Worten: die Kinder und Jugendlichen, ihre Familien und sozialen Gruppierungen müssen auf diese Entwicklung vorbereitet und zu mehr Selbständigkeit und Eigenverantwortung befähigt werden. An die Stelle eines Sozialstaates, der die soziale Sicherheit vor allem durch monetäre Transferleistungen zu gewährleisten meinte, tritt nun eine öffentliche Verantwortung, die die Voraussetzung dafür schafft, dass die Menschen selbständig und eigenverantwortlich handeln und ihre Probleme lösen können.

Wer ist denn nun der Träger dieser öffentlichen Verantwortung? In einer pluralistischen Gesellschaft sind dies: - der soziale Nahraum der Kinder und Jugendlichen, insbesondere die Netzwerke der Familien,

- die Vielzahl der interessenorganisierenden, mitgliedergesteuerten Vereine und Verbände,

- die Wirtschaftsunternehmen, die nicht nur über Produktion und Distribution von Gütern bestimmen, sondern auch über Ausbildung und Arbeit,

- die Arbeitgeber- und Arbeitnehmerorganisationen, die die Politik beeinflussen,

- die »/dealvereine», d. h. ideengesteuerte Organisationen, wie insbesondere die Kirchen, die Umweltschutzverbände, Amnesty International, Greenpeace u.a.m.,

- die Massenmedien, die sowohl die Politik wie die Bürgerinnen und Bürger beeinflussen,

- die Gemeinden und Kreise, die die örtliche Gemeinschaft organisieren, und schließlich

- der Staat, d. h. Bund und Länder, die letztlich die Verantwortung dafür tragen, dass das System funktioniert, und die subsidiär die öffentliche Verantwortung gewährleisten müssen.

Trotz allen Geredes von der »Entstaatlichung des Staates" muss man daran festhalten, dass der Staat die öffentliche Verantwortung für das Aufwachsen von Kindern und Jugendlichen gewährleisten muss. Im traditionellen Sozialstaat musste der Staat das so genannte Existenzminimum der Menschen gewährleisten, d. h. im Klartext den Sozialhilfesatz, egal ob sich dieser nach einem fiktiven Warenkorb oder nach einem prozentualen Anteil des Durchschnittseinkommens bemisst. Öffentliche Verantwortung heißt dagegen, dass der Staat eine Selbsthilfefähigkeit gewährleisten - und schlimmstenfalls zahlen - muss.

Für diese "gemischte Gesellschaft « wurde in der Sozialpolitik der Ausdruck "Welfare mix « erfunden, weil es sich um staatliche, kommunale, freie gemeinnützige und kommerzielle Träger handelt. Von staatlichen und kommunalen Trägern kann man in der Tat die Übernahme öffentlicher Verantwortung für das Aufwachsen von Kindern und Jugendlichen erwarten, auch von gemeinnützigen Trägern, denn das Steuerrecht bindet die an sich freien Träger an die Erfüllung öffentlicher Aufgaben. Kann man aber auch kommerziellen Trägern, wie z. B. Wirtschaftsunterneh- men, die Übernahme öffentlicherVerantwortung zumuten? Vielleicht ist es an der Zeit, wieder einmal daran zu erinnern, dass im Grundgesetz der Satz steht: „Eigentum verpflichtet. Sein Gebrauch soll zugleich dem Wohle der Allgemeinheit dienen «. Dieser Ausdruck »öffentliche Verantwortung « trifft die deutsche staatspolitische Lage besser als die deutschen Übersetzungen von "civil society « und "communitarianism «, die aus den Vereinigten Staaten importiert wurden, die bekanntlich so etwas wie "Staat " gar nicht kennen, sondern nur »civil society«, »community« und "government«.

Im Bereich der Kinder- und Jugendhilfe besteht eine solche gemischte öffentliche Verantwortung bereits, denn neben kommunalen Trägern gibt es die Wohlfahrts- und Jugendverbände als Träger, aber auch kommerzielle Leistungsanbieter, alle eingebunden in ein System lokaler Planung und staatlicher Aufsicht. Der Elfte Kinder- und Jugendbericht enthält eine ausführliche Beschreibung und Bewertung von Organisationen und Verfahren der Kinderund Jugendhilfe und empfiehlt neben zahlreichen Verbesserungen im Wesentlichen die Beibehaltung dieser Form öffentlicher Verantwortung. Er regt allerdings die Einführung eines fachlich kontrollierten Wettbewerbs im Rahmen einer staatlich-kommunalen Planung und Aufsicht an, d. h. den Rückzug der Kommunen aus der Trägerschaft der Einrichtungen. An dieser Stelle können diese Vorschläge nicht im Einzelnen erläutert werden, es soll jedoch darauf hingewiesen werden, dass andere Instanzen, die öffentliche Verantwortung für das Aufwachsen von Kindern und Jugendlichen tragen, wie z. B. das Schulwesen, von einer solchen Organisationsform noch meilenweit entfernt sind.

Die Kommission hat auf der Grundlage der empirischen Forschung eine umfassende Analyse der Lebenslagen von Kindern und Jugendlichen vorgelegt und jeweils Empfehlungen nicht nur für die Kinder- und Jugendpolitik, die Kinder- und Jugendhilfe, sondern auch für andere Politik- und Verwaltungsbereiche angegeben (vgl. BMFSFJ 2002, Teil B). Sie legt darüber hinaus die folgende allgemeine Begründung einer neuen Kinder- und Jugendpolitik vor:

"Die Kommission versteht die Jugendpolitik als >Lebenslagenpolitikく. Sie meint damit eine Politik im Interesse von Kindern und Jugendlichen, die erstens von den objektiven sozialen Unterschieden in den Lebenslagen von Kindern und Jugendlichen ausgeht und sich auf diese richtet, nämlich insbesondere auf die Unterschiede nach Geschlecht, Bildung, Schicht bzw. Klasse, Region, Migration und Alter, die zweitens die subjektive persönliche Lebensführung der Kinder und Jugendlichen berücksichtigt, nach den Einstellungen und Handlungsmöglichkeiten der Kinder und Jugendlichen selber fragt und ihre Selbständigkeit stärkt und die drittens den Auftrag der Kinder- und Jugendhilfe nach $\S 1 \mathrm{KJHG}$ ernst nimmt, wonach die Kinderund Jugendhilfe zur Schaffung positiver Lebensbedingungen für Kinder und Jugendliche und ihre Familien beizutragen hat « (BMFSFJ 2002, S. 51). 
Eine so verstandene Lebenslagenpolitik findet immer dort statt, wo öffentliche Verantwortung für das Aufwachsen von Kindern und Jugendlichen in allen Lebensbereichen der jungen Generation übernommen wird. »In der Kinder-und Jugendhilfe findet sie schließlich darin ihren Ausdruck, dass sie für alle jungen Menschen Leistungen erbringt und Chancengerechtigkeit durch individuelle Förderung, Beratung und Hilfe sowie Vernetzung und Koordination vor Ort unterstützt« (ebd., S. 248).

Zum Schluss soll nochmals der Begriff der Verantwortung - hier aber nur bezogen auf die öffentliche Verantwortung - aufgegriffen werden, so wie er oben durch seine fünf Handlungselemente gekennzeichnet wurde. Und es wird danach gefragt, ob der Staat seine Aufgabe, öffentliche Verantwortung zu tragen, in Form staatlicher Tätigkeit und durch die Gewährleistung von Selbsthilfefähigkeit der gesellschaftlichen Akteure, annähernd erfüllt. Die Beispiele sind dafür aus dem Elften Kinder-und Jugendbericht entnommen.

- Aufgaben erkennen: Einige der heute anstehenden Aufgaben für das Aufwachsen von Kindern und Jugendlichen hat die Politik erkannt, z. B. die Schaffung von Ganztagsbetreuungsangeboten für alle Kinder; andere dagegen nicht, z. B. den Kampf gegen die zunehmende soziale Segregation.

- Pflichten erfüllen: Pflichten gegenüber der jungen Generation werden häufig auf Druck erfüllt, z. B. auf Druck durch das Bundesverfassungsgericht in Karlsruhe, wie im Falle des Rechts auf einen Kindergartenplatz oder auf einen gerechten Familienlasten- und -leistungsausgleich; andere Pflichten bleiben unerfültt, wie z. B. die von der Bundesregierung auf dem Europäischen Beschäftigungsgipfel 1997 übernommene Ausbildungsund Beschäftigungsgarantie für alle Jugendlichen.

- Rechenschaft ablegen: Die Kinder- und Jugendberichterstattung durch eine unabhängige Sachverständigenkommission ist eine Form, Rechenschaft abzulegen. Der Begriff "Verantwortung « enthält das Wort »Antwort«, und die Öffentlichkeit hat ein Recht auf eine Antwort auf die gestellten Fragen, man sollte sich nicht damit zufrieden geben, dass Regierungen nur allzu häufig sich darauf beschränken, ihre Politik zu rechtfertigen.

- Für Kinder- und Jugendliche einstehen: Kinder und Jugendliche kritisieren zu Recht, dass die Erwachsenen selber nicht nach den Grundsätzen leben, zu denen diese sie erziehen wollen. Wie kann man in einer Welt voller Gewalt zur friedlichen Konfliktregelung erziehen, wie in einer Welt voller Drogen zur Abstinenz? Der Kinderund Jugendschutz muss mit dem Grundwiderspruch leben, dass Kinder und Jugendliche vor dem bewahrt werden sollen, was für sie den Inbegriff des Erwachsenenseins ausmacht.

- Für Versagen haften: In unserem ersten Einleitungsfall, "Eltern haften für ihre Kinder«, ging es um Schadensersatz und Strafe; doch dieser Fall wurde für die Beschreibung der »öffentlichen Verantwortung " für das Aufwachsen von Kindern und Jugendlichen nicht leitend, da die Politik selten für Versagen haftet. Hans Jonas schreibt in seinem Buch »Das Prinzip Verantwortung«, dass die Haftung des Staatsmannes für seine Politik im Falle des Versagens im Rücktritt oder in der Abwahl bei der nächsten Wahl besteht.

\section{Anmerkung}

1 Überarbeitete Fassung eines Vortrags auf dem 18. Kongress der DGFE »Innovation durch Bildung" in München am 26.03.02

\section{Literatur}

Bundesministerium für Familie, Senioren, Frauen und Jugend (BMFSF); Hrsg.): Elfter Kinder- und Jugendbericht. Bericht über die Lebenssituation junger Menschen und die Leistungen der Kinder- und Jugendhilfe in Deutschland. Bundestagsdrucksache 14/8181. Berlin 2002

Jonas, Hans: Das Prinzip Verantwortung. Frankfurt am Main 1979 